\title{
Simulation of Mixing Processes by Means of Mesh-free Particle Approach
}

\author{
C. Kussmann, P. Drage
}

qpunkt $\mathrm{GmbH}$, Graz, Austria

E-mail: christian.kussmann@qpunkt.at (C. Kussmann)

Sci Pharm. 2010; 78: 667

doi:10.3797/scipharm.cespt.8.PMS40

This paper presents results of the simulation of unsteady mixing processes using a unique, mesh-less particle approach. Similar simulations, performed by Hörmann et.al [1] using common Computational Fluid Dynamics (CFD) have already been published in the past. The new method applied offers computational possibilities for problems involving transient dynamics, free surface analysis, moving boundaries or fluid-structure interaction. The simulations performed within the framework of this project are based on the Navier Stokes Equations using Large Eddy or Detached Eddy approaches. Turbulent energy dissipation models are used to resolve scales which are not captured by the particle resolution.

The investigations presented focus on the reproduction flow phenomena in a mixing tank, including viscosity adaption due to particle dissolution and analysis of particle concentration using a Discrete Phase Model (DPM). Applying CFD packages, problems including moving boundaries require time consuming workarounds, which often lead to errors or divergence of the simulation. Due to the used mesh-less method, only the physical and mechanical properties of the problem, like number of revolutions, filling height or initial fluid viscosity need to be defined.

The results of the simulation are compared with Particle Image Velocimetry (PIV) measurements, showing good agreement.

[1] Hörmann Th, Redlinger-Pohn J, Schaffer M, Suzzi D, Radl S, Khinast JG. Simulation of mixing processes for the manufacture of pharmaceutical products. International Graz Congress for Pharmaceutical Enginering 2009. 